\title{
Analysis of Influence of Coil Gradient System on Vibration Properties and Acoustic Noise Level Generated by the Low Field MRI Device
}

\author{
Jiří Přibil ${ }^{1}$, Anna Přibilová2 ${ }^{2}$ Ivan Frollo ${ }^{1}$ \\ ${ }^{1}$ Institute of Measurement Science, SAS, Dúbravská cesta 9, SK-84104 Bratislava, Slovakia, Jiri.Pribil@savba.sk \\ ${ }^{2}$ Institute of Electronics and Photonics, FEE\&IT, SUT, Ilkovičova 3, SK-812 19 Bratislava, Slovakia
}

\begin{abstract}
The paper focuses on investigation of influence of the volume inserted in the scanning area of the magnetic resonance imaging (MRI) device working with a low magnetic field generated by a pair of permanent magnets on vibration and acoustic noise. In addition, its aim is to evaluate the influence of different types of used scan sequences, different settings of slice orientation and scan parameters on the energy and spectral properties of vibration and noise generated by the gradient coil system of the MRI device. Two basic measurements were performed within this work: mapping of sound pressure levels in the MRI device vicinity and parallel acquisition of vibration signals by sensors mounted on the lower and upper parts of the MRI gradient system. The paper next analyzes changes in properties of the vibration signals for the examined person lying in the scanning area compared with the situation of using only the testing phantom. Spectral characteristics of the recorded vibration signals are then analyzed statistically, and compared visually and numerically. The obtained results of the detailed analysis will be used for improvement of noise suppression algorithms applied to a speech signal recorded simultaneously with scanning of the human vocal tract for its $3 \mathrm{D}$ modeling.
\end{abstract}

Keywords: Vibration and acoustic noise measurement, magnetic resonance imaging, signal processing, statistical analysis.

\section{INTRODUCTION}

Magnetic resonance imaging (MRI) scanners working with a weak magnetic field are widely used as tools for noninvasive imaging of different parts of a human body in modern clinical practice. These scanners are also powerful tools in the research of weak magnetic materials that can be used as composites of various body or dental implants. The core of the MRI device is the gradient coil system creating three orthogonal linear magnetic fields in a static magnetic field. The MRI scanners working with low static magnetic field $B_{0}$ strength up to $0.2 \mathrm{~T}$ can use a pair of permanent magnets typical for the open-air MRI device type [1] or a resistive water-cooled magnet in the whole-body MRI device. Three gradient fields for selection of slices of tested samples in three orthogonal Cartesian directions are created by the currents flowing in the conductors of the gradient coils. These currents in the presence of the static magnetic field result in large oscillating Lorentz forces and subsequent gradient coil conductor vibration giving rise to acoustic noise [2], [3]. The acoustic properties of the gradient system can be characterized by the frequency response function [4]. Although there exist methods for fault diagnosis of mechanical systems that are based on recognition of acoustic signals [5], the vibration measurement gives better insight into the origin of this acoustic noise.

Recent scientific publications in this area have dealt with detailed modeling and simulation of gradient coils including theoretical description of Lorentz forces [3] and a phenomenon called Lorentz mechanical damping [6] for each of the turns of standard coils as well as of special coils used in the gradient system of the MRI device [7]. On the other hand, gradient coils can be modeled as one object [8]. In accordance with our previous research on measurement, mapping, and analysis of noise and vibration produced by the gradient system [9], [10], the coil is considered to be one unit in the present work.

The original contribution of this paper lies in investigation and comparison of vibration and noise conditions in two parts of the MRI gradient system - upper and lower gradient coils. They both are characterized by a similar magnetic flux density differing in its orientation due to different location of the gradient coils with respect to the permanent magnet (under or above it). Excited vibrations in upper and lower parts of the gradient system during execution of the MR scan sequence are evaluated simultaneously with regard to the mass of the examined object lying on the cover of the lower gradient coils. In our previous studies we analyzed only the 
lower gradient coils of the MRI device from the viewpoint of noise and vibration generation and their signal properties. It was here extended to measure intensity, distribution, and spectral properties of mechanical vibration and acoustic noise produced by both parts of the gradient system of the low magnetic field MRI device.

The paper describes measurements performed in the scanning area and in the neighborhood of the open-air MRI equipment. Measurements were carried out separately for each of the two parts and the obtained results were compared. First, detailed mapping of the noise sound pressure level (SPL) was performed in the vicinity of the MRI scanner. Then, parallel real-time recording of the vibration signals on both parts of the MRI gradient system was carried out. These signals were subsequently processed off-line to determine basic properties and spectral features. Next, the obtained results were compared visually and numerically. The main comparison experiment consisted of the analysis of influence of the mass of a tested person/object in the scanning area during execution of the MR scan sequence on intensity and spectral properties of the vibration signal. In addition, the impact of different parameters and types of used MR scan sequences on the vibration signal production was analyzed.

\section{SUBJECT \& METHODS}

As mentioned above, the MRI devices are primarily used in the clinical diagnostic practice to scan various parts of a human body or biological tissues [11]. Therefore, the designation of $x, y$, and $z$ axes to select slices of a tested subject originates from the names of three body planes used in medical terminology [12]. The coronal (frontal) plane is a vertical plane dividing the body into ventral (anterior) and dorsal (posterior) portions; the sagittal plane divides it vertically into left and right sides; the transverse (crosssectional) plane is a horizontal plane dividing the body of the examined person into superior (upper) and inferior (lower) sections. The gradient system of the latter ones consists of $2 \times 3$ planar coils situated between the magnets and the RF receiving/transmitting coil with the tested object/subject as documented by the photo in Fig.1.a) and the principal schematic layout in Fig.1.b).

Even though every scanning MR sequence enables user settings of the orientation parameter, in the majority of cases it has a predefined value for the best image acquisition. Different orientation brings use of different $x, y$, and $z$ gradient coils during the execution of the scan sequence. It has also impact on generated vibration and subsequently propagated acoustic noise.

The second parameter having a great influence on the intensity of the produced vibration and noise is the volume size of the tested object/subject in the scanning area of the MRI tomograph. According to the physical principle of MRI [14], the larger the volume of the tested sample, the higher will be the electrical current flowing through the gradient coils in order to choose $3 \mathrm{D}$ coordinates by the equivalent change in the magnetic field. On the other hand, the field of view (FOV) does not depend on the size of the sample. If the spatial resolution and the sampling time remain unchanged, the gradient field decreases in the final effect. Generally, higher gradient coil current results in stronger magnetic field producing stronger Lorentz forces which in turn causes stronger vibration signal and its different spectral properties. From the vibroacoustic point of view, the tested person/sample/phantom placed on the patient's bed changes the overall mass, stiffness, and damping of the whole scanning system, mainly that of the lower gradient coil structure.

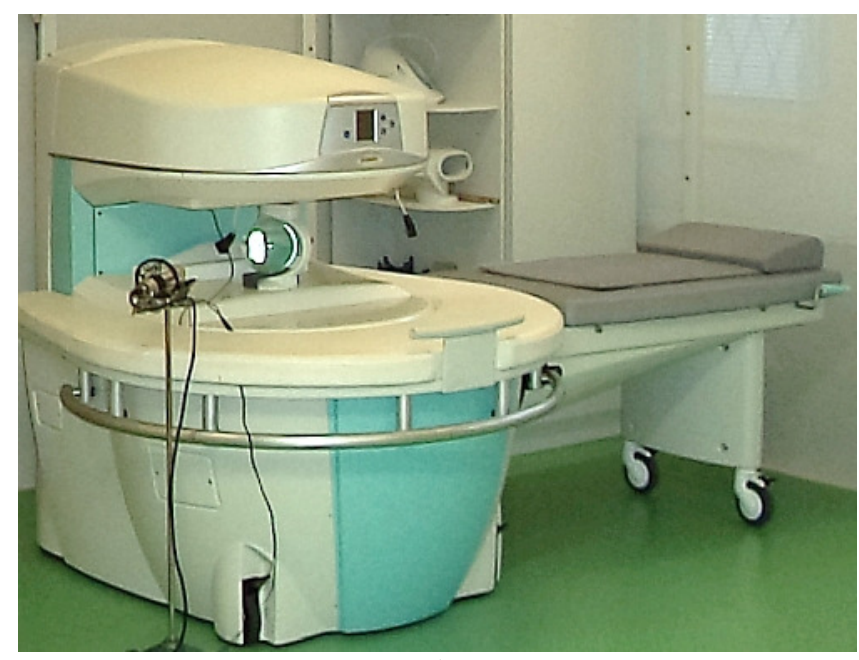

a)

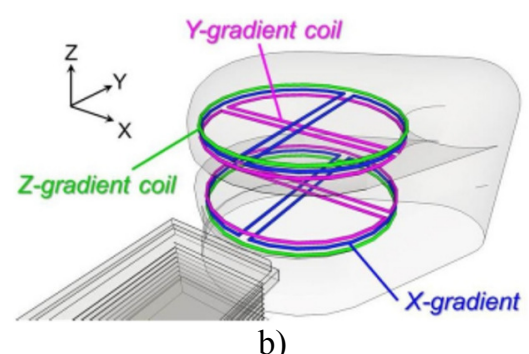

Fig.1. a) Illustration photo of the open-air MRI device [1], b) principal $3 \mathrm{D}$ schematic plan of the gradient system [13].

A lot of methods may be used to determine the energy of the signal. Two of them using the vibration signal in a defined region of interest (ROI) are performed by calculating:

- the root mean square (RMS) of the signal,

- the magnitude of the power spectrum $|S(k)|$ of the vibration signal $s(n)$ using $N_{F F T}$-point FFT. The signal is processed in frames of duration dependent on the basic vibration frequency $F_{\mathrm{V} 0}$ (similar to the fundamental frequency of the speech signal) and the frame energy is estimated using the autocorrelation coefficient $r_{0}$ as

$$
E n_{r 0}=\frac{2}{N_{F F T}} \sum_{k=1}^{N_{F F r} / 2}|S(k)|^{2}
$$

or by the first cepstral coefficient $c_{0}$ as

$$
E n_{c 0}=\sqrt{\left[\prod_{k=1}^{N_{F F T} / 2}|S(k)|^{2}\right]^{\frac{2}{N_{F F T}}}} .
$$


Comparison of vibration sensors for measurement in the weak magnetic field environment

In general, it is important that the vibration sensor has good sensitivity and a maximally flat frequency response. The sensor's frequency range must encompass the relevant partial frequencies of the measured vibration signal [15]. They are concentrated in the low band due to the frequency-limited gradient pulses [16]. Due to similar frequency range covered by bass audio signals, the sensors constructed for acoustic pickup in musical instruments can be used for vibration signal measurement and recording. In our previous works, we used the Cejpek SB-1 sensor primarily designed for contrabass sound pickup with a piezoelectric element mounted on the circular $0.25 \mathrm{~mm}$ thin $1 "$ brass disc [9], [10]. For the purpose of the experiments presented here, it is necessary to measure parallel vibration of the lower and the upper set of the gradient coils. The requirements for use in the low $B_{0}$ environment are fulfilled also by another piezoelectric sensor for musicians - the Shadow SH-SB2 Double Bass Pickup with two $0.5 \mathrm{~mm}$ thin $20 \mathrm{~mm}$ disc transducers, further called as " $S B-2 a, b$ ". Its first usage must be preceded by a calibration procedure and a measurement of sensitivity and frequency response:

$$
G_{a \mathrm{~dB}}(f)=20 \cdot \log _{10}\left(B_{a}(f) / B_{a}\left(f_{\text {ref }}\right)\right),
$$

where $B_{\mathrm{a}}$ represents sensitivity of the accelerometer in $\left[\mathrm{mV} / \mathrm{m} \mathrm{s}^{-2}\right]$, and $f_{\text {ref }}$ is the reference frequency.

Our calibration experiment was carried out using the Brüel \& Kjær beat frequency oscillator with the output power amplifier and the vibration exciter ESE 201. As a reference sensor, the accelerometer KD35a from the company Metra Mess-und Frequenztechnik was used. This standardized accelerometer has a guaranteed sensitivity and the frequency measurement range from $20 \mathrm{~Hz}$ to $10 \mathrm{kHz}$. The sensors were mounted on a plate of the vibration exciter - see the detailed photo in Fig.2.

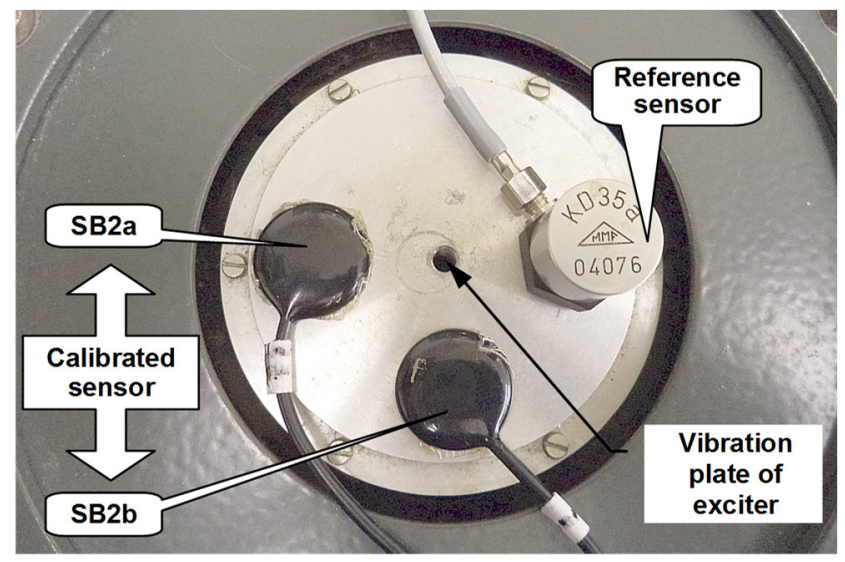

Fig.2. Photo of practical mounting of the sensors on the plate of the vibration exciter used in the calibration experiment.

The output voltage for supply of this exciter within the selected frequency range was measured by the LFmillivoltmeter and corrected manually. Electrical signals from the vibration sensors were measured simultaneously by a two-channel digital oscilloscope. Two types of parameters of vibration sensors were measured and compared:

- relative sensitivity at the reference frequency of $125 \mathrm{~Hz}$,

- frequency response in the range of $<20-2000 \mathrm{~Hz}>$ at the chosen input exciter voltage $U_{\mathrm{EXC}}=360 \mathrm{mV}$.

The obtained dependence of the output voltage (corresponding to the sensor's sensitivity in $\left[\mathrm{mV} / \mathrm{m} \mathrm{s}^{-2}\right]$ ) on the excitation voltage for the tested sensors, and the measured frequency response are shown in Fig.3.a), Fig.3.b).
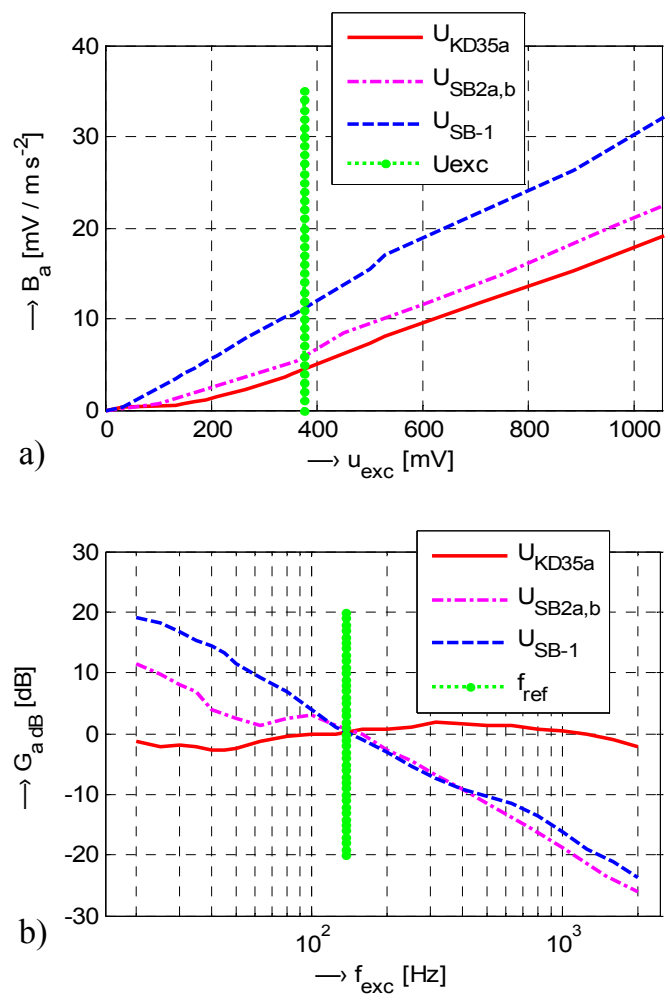

Fig.3. Measured sensitivity characteristics for the tested sensors at $f_{\text {ref }}=125 \mathrm{~Hz}, U_{E X C}=360 \mathrm{mV}$ a); sensors' frequency responses $G_{a}$ dв in the range of $<20-2000 \mathrm{~Hz}>$ b).

\section{EXPERIMENTS AND RESULTS}

This study comprises two basic measurement and comparison experiments. The first part of the performed experiments was focused on the measurement of acoustic noise levels in the vicinity of the scanning area of the openair MRI equipment E-scan Esaote Opera [1]. The second part consists of experiments based on the vibration signals recorded inside the scanning area of the investigated MRI device. The stored records are next processed for evaluation and comparison of the vibration signal properties.

In the first-step experiments, the effect of the parameters of the MR scan sequences on the acoustic noise and the vibration was analyzed. The measurements were carried out for different scan sequences by setting of $T_{\mathrm{SEQV}}=\{$ SpinEcho $\mathrm{SE}$, GradientEcho - GE\}, different orientation of scan slices choosing $T_{\text {ORIENT }}=\{$ Coronal, Sagittal, Transversal $\}$, and different setting of TE parameter $T_{\mathrm{TE}}=\{16,22,26\} \mathrm{ms}$. 
The main experiments were focused on the analysis of the influence of the mass and the volume size of the inserted subject in the scanning area on the produced:

a) level of the acoustic noise (SPL measurement),

b) energy and spectral properties of the vibration of the MRI device.

This task was practically realized under two conditions: the testing spherical phantom placed in the RF scan coil and the testing person lying in the MRI device.

Taken vibration signals were next processed: calculated periodograms were compared visually and the basic and supplementary spectral features were analyzed. It means the signal energy, spectral density, its envelope, spectral tilt, harmonic to noise ratio (HNR), first two dominant frequencies $F_{\mathrm{V} 1}, F_{\mathrm{V} 2}$ of the vibration and their mutual positions were determined. Within the subsequent statistical analysis the histograms of supplementary spectral properties (spectral centroid, spectral entropy, etc.) were calculated see the block diagram in Fig. 4.

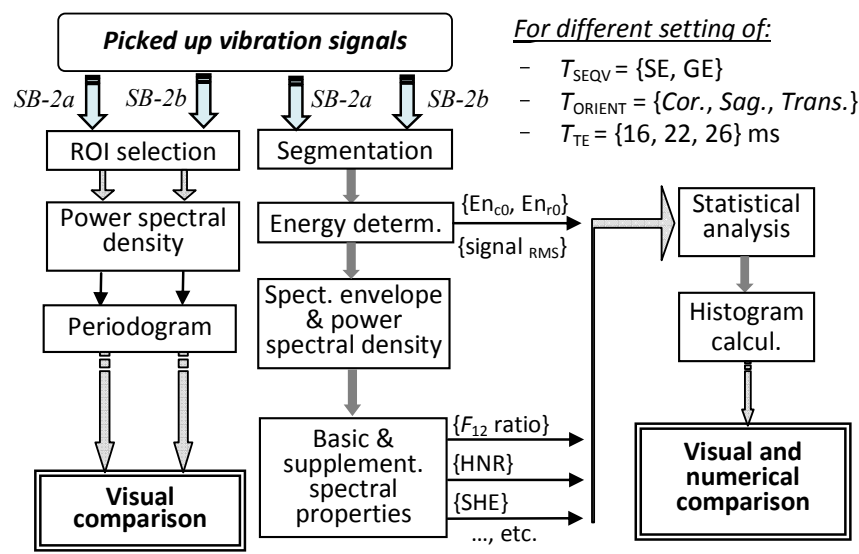

Fig.4. Block diagram of vibration signal analysis and comparison.

The baseline measurement was carried out during the execution of 3D and Hi-Resolution (Hi-Res) sequences useful for scanning of the human vocal tract [17], [18]. For this comparison experiment the following setting of the MR scan sequence was used: Hi-Res SE HE scan sequence (echo time $\mathrm{TE}=18 \mathrm{~ms}$, repetition time $\mathrm{TR}=500 \mathrm{~ms}$ ), sagittal orientation. For comparison with using of the GE sequence (Hi-Res GE 16), the scan parameters were: $\mathrm{TE}=16 \mathrm{~ms}$, $\mathrm{TR}=400 \mathrm{~ms}$, sagittal orientation. In all cases the imagining parameters $\mathrm{FOV}=270 \mathrm{~mm}$, the resolution of $192 \times 192$ samples, and the slice thickness $=4.7 \mathrm{~mm}$ were applied.

\section{A. Measurement of the acoustic noise SPL distribution}

The MRI device includes also an adjustable bed with continual positioning in the range of 180 degrees with the initial direction at the left corner near the temperature stabilizer device. Therefore, the detailed measurement of the directional pattern of the acoustic noise SPL distribution in the MRI device vicinity in the range of $0 \sim 180$ degrees with 15 degree steps was carried out first. The sound level meter (multi-function environment meter Lafayette DT 8820) was placed at the distance of $60 \mathrm{~cm}$ from the central point of the scanning area. The measurement was carried out with the sound level meter placed in three heights between the upper and lower plastic covers of the gradient coils as documented by the arrangement photo in Fig.5.:

a) on the level "A" (bottom gradient coils); at the height of $75 \mathrm{~cm}$ from the floor),

b) on the level "B" (in the middle between both parts of the gradient system); $85 \mathrm{~cm}$ from the floor,

c) on the level "C" (upper gradient coils); $95 \mathrm{~cm}$ from the floor.

A separate measurement of the mere background noise (mostly due to the temperature stabilizer) was additionally performed to compare values with the ones obtained during the execution of the MR scan sequence.

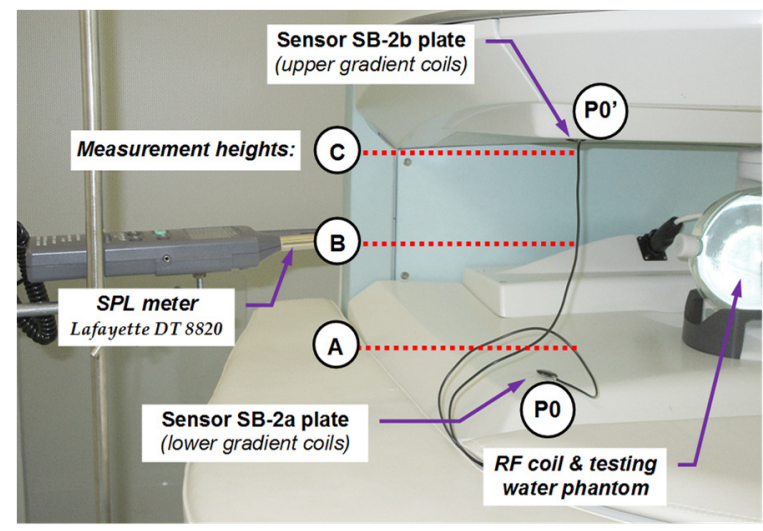

Fig.5. Arrangement photo of SPL noise measurement in the device vicinity and parallel recoding of vibration signals on upper and lower plastic covers of the gradient coils of the MRI Opera.

The measured directional patterns of the noise source for three sound level meter heights, including the background noise $S P L_{0}$, are presented in Fig.6.

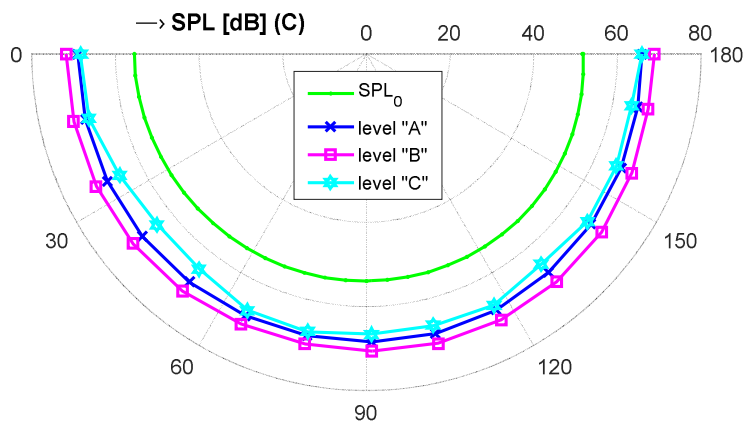

Fig.6. Resulting directional pattern of the SPL noise distribution measured in heights "A", "B", and "C" together with the background noise $S P L_{0}$; SE sequence, coronal slice orientation.

The next analysis was performed to measure SPL values of the noise at the height "B" for different $T_{\mathrm{SEQV}}$ and $T_{\text {ORIENT }}-$ see graphical comparison in Fig.7. For all these described measurements the spherical testing phantom was placed in the knee RF coil. 


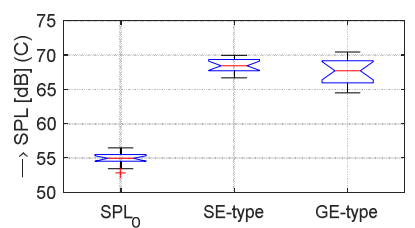

a)

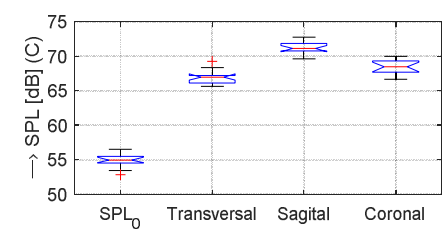

b)
Fig.7. Basic statistical parameters of noise SPL including the $S P L_{0}$ values for: two scan sequences using the coronal orientation a), three slice orientations using the SE scan sequence b); measured at the height of $85 \mathrm{~cm}$ in the direction of 30 degrees.

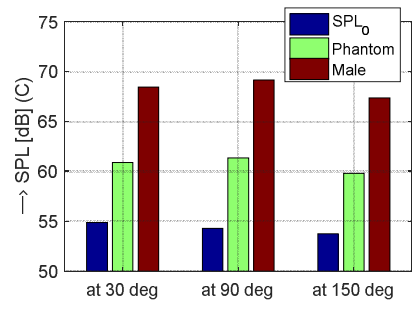

a)

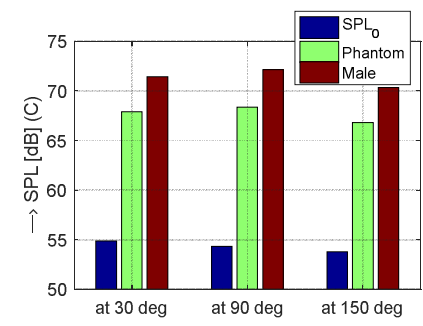

b)
Fig.8. Mean SPLs together with the background noise $S P L_{0}$ for the water phantom and the tested male person: measured at heights of $75 \mathrm{~cm} \mathrm{a}$ ), and $95 \mathrm{~cm} \mathrm{~b})$; in the direction $\{30,90,150\}$ degrees.
Finally, the noise SPL was measured at two heights " $A$ " and "C" separately in the directions $N_{\text {DIR }}=\{30,90$, and 150$\}$ degrees for different objects placed in the scanning area (phantom/person) - the mean values are presented in the form of the bar-graphs in Fig.8.

\section{B. Analysis of vibration signals recorded in the MRI device}

For recording of the vibration signals inside the scanning area of the MRI device the SB-2 sensors were applied. The sensing discs were mounted on the surface of the plastic holders of the gradient coils: $S B-2 a$ on the lower plate $(\mathrm{Low} / \mathrm{L}), S B-2 b$ on the upper plate $(\mathrm{Up} / \mathrm{U})$, both in the direction of 45 degrees (left corner) - practically at the positions P0/P0', as shown in Fig.5. If not stated otherwise, the signals were taken at these positions during execution of the SE sequence with the basic setting.

As a preliminary comparison, the difference between the signals recorded by two sensors separately $(S B-2 a / S B-2 b)$ versus the sum signal obtained by parallel connection of both discs $(S B-2 a+b)$ was investigated - see the graphical comparison in Fig.9. The auxiliary measurements contain investigation of the influence of different settings of $T_{\mathrm{SEQV}}$, $T_{\text {ORIENT, and }} T_{\mathrm{TE}}$ on spectral properties of the vibration signal - see the graphical comparison by the set of graphs in Fig.10., Fig.11., and Fig.12. In both previous experiments the vibration signal was recorded using the testing phantom.
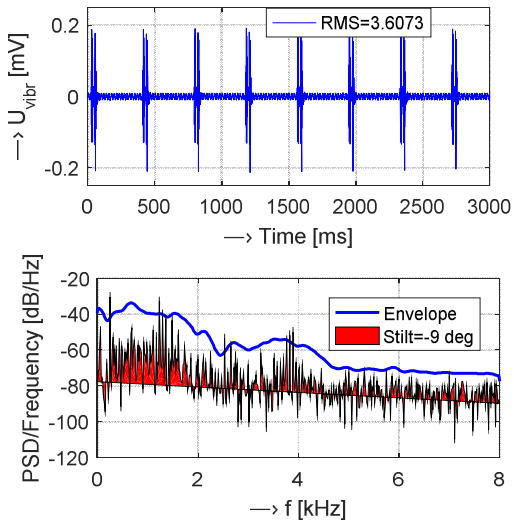
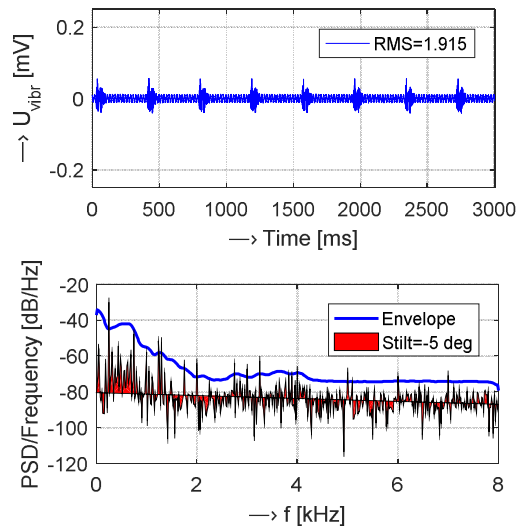
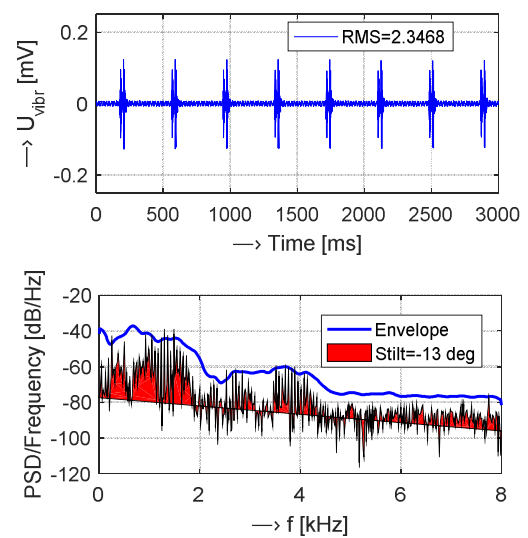

Fig.9. Comparison of signals including their RMS (upper graphs) and periodograms with smoothed spectral envelopes including the spectral tilt (bottom graphs) for three types of vibration signals picked up by: $S B-2 a$ (left), $S B-2 b$ (middle), and $S B-2 a+b$ (right).

The main experimental recording of the vibration signals was performed using two types of the testing spherical phantoms filled with doped water, with different diameters and volumes inserted in the knee RF scan coil: the greater one with the diameter of $140 \mathrm{~mm}$ and the weight of $0.75 \mathrm{~kg}$ (next called "WP1"), and the smaller one with the diameter of $85 \mathrm{~mm}$ and the weight of $0.5 \mathrm{~kg}$ ("WP0") as documented by the left photo in Fig.13.a). In the next step the vibration signals were recorded while the tested person was lying in the patient's bed of the MRI device (one male and one female person with the weights approx. 75 and $55 \mathrm{~kg}$, next called "M1/F1") - see the right photo in Fig.13.b). The obtained mean values of the vibration signal energy using two types of the testing phantoms together with the values for the lying persons are numerically presented in Table 1. and graphical comparison of spectral properties is shown by the set of graphs in Fig. 14.

Table 1.: Comparison of mean energy values of vibration signals for different objects placed in the scanning area of the MRI device.

\begin{tabular}{|c|c|c|c|c|c|c|}
\hline \multirow{2}{*}{$\begin{array}{c}\text { Subject } \\
\text { type }\end{array}$} & \multicolumn{3}{|c|}{ Signal of $U_{\text {SB-2a }}$ (lower) } & \multicolumn{3}{|c|}{ Signal of $U_{\text {SB-2b }}$ (upper) } \\
\cline { 2 - 7 } & $\mathbf{R M S}^{\text {A) }}$ & $\boldsymbol{E n}_{\mathbf{c} 0}$ & $\boldsymbol{E n}_{\mathbf{r} 0}$ & $\mathbf{R M S}^{\mathrm{A})}$ & $\boldsymbol{E n}_{\mathbf{c} 0}$ & $\boldsymbol{E n}_{\mathbf{r} 0}$ \\
\hline WP0 & 7.00 & 1.031 & 6.916 & 4.21 & 0.746 & 6.248 \\
\hline WP1 & 6.92 & 0.998 & 6.814 & 4.36 & 0.824 & 6.312 \\
\hline M1 & 5.35 & 1.006 & 6.905 & 10.10 & 0.887 & 6.612 \\
\hline F1 & 5.73 & 1.005 & 6.739 & 8.63 & 0.862 & 6.608 \\
\hline
\end{tabular}

A) Calculated from the ROI with a duration of 3 seconds. 

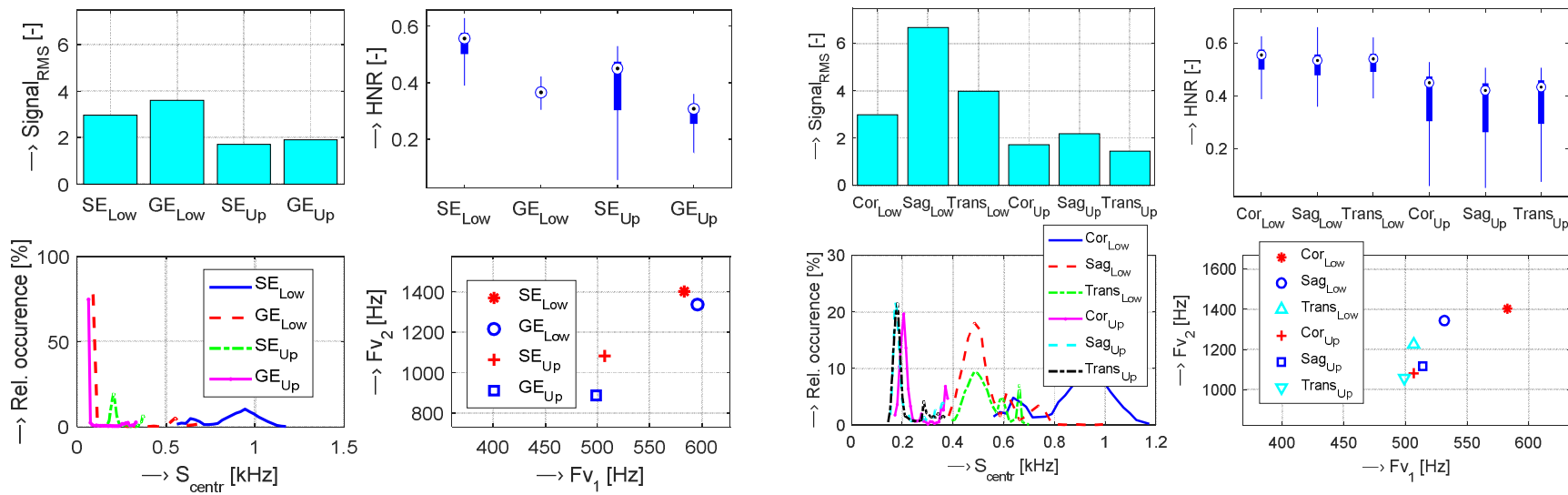

Fig.10. Comparison of the vibration energy and spectral properties \{signal ${ }_{\mathrm{RMS}}, \mathrm{HNR}$, spectral centroid, $F_{\mathrm{V} 1}-F_{\mathrm{V} 2}$ graph of the signals recorded in parallel from the sensor's plates $S B-2 a$ (Low), $S B-2 b$ (Up) for the scan sequences $S E / G E$.
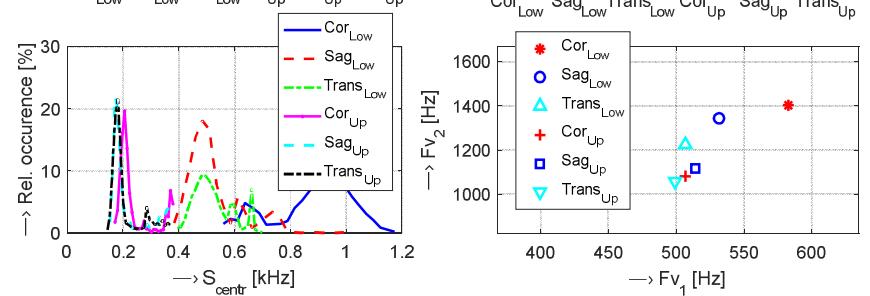

Fig.11. Comparison of the vibration energy and spectral properties \{signal $\mathrm{RMS}, \mathrm{HNR}$, spectral centroid, $F_{\mathrm{V} 1}-F_{\mathrm{V} 2}$ graph \} of the signals recorded in parallel from the sensor's plates $S B-2 a$ (Low), $S B-2 b$ (Up) for the slice orientations Coronal, Sagittal, and Transversal.
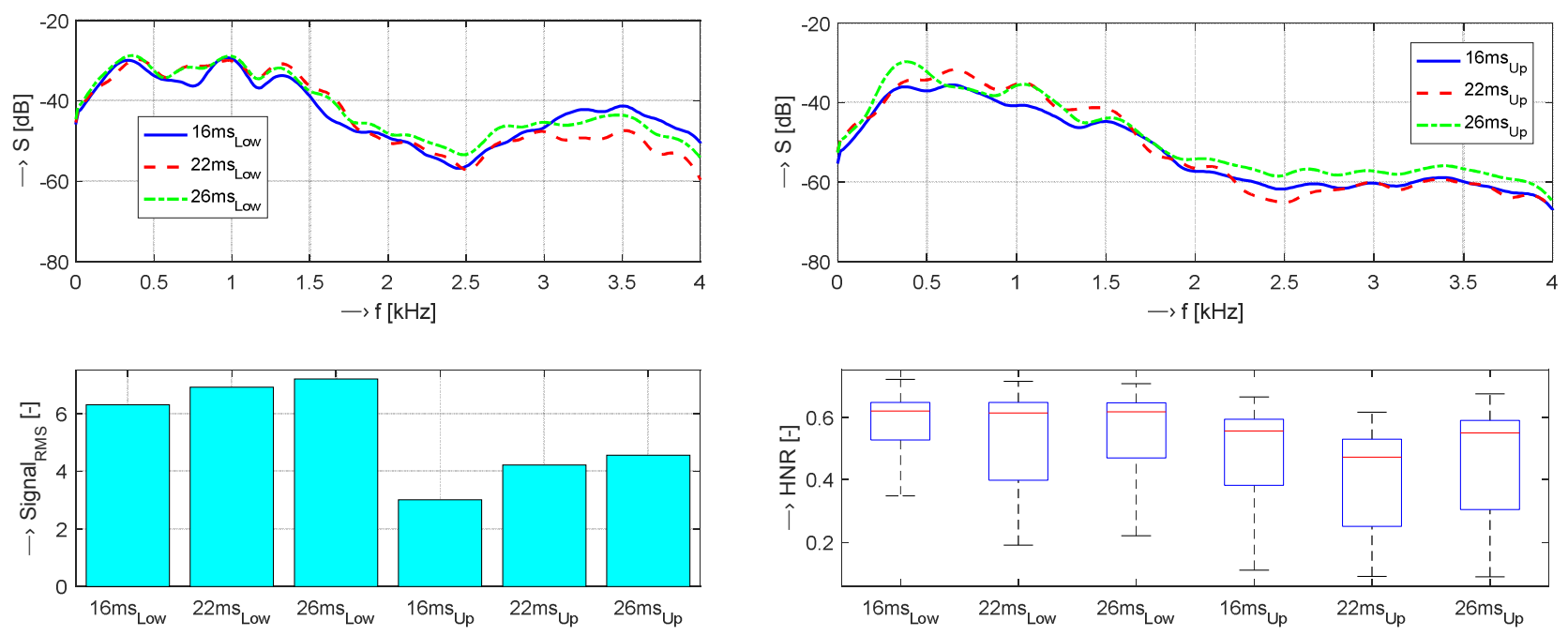

Fig.12. Comparison of vibration signal properties taken from the sensor's plates $S B-2 a$ and $S B-2 b$ using TE $=\{16,22,26\}$ ms: spectral envelopes in the low frequency range $0-4 \mathrm{kHz}$ (upper graphs), mean signalRMS, and a box-plot of basic statistical properties of HNR (lower graphs); used GE sequence, sagittal orientation, WP1.

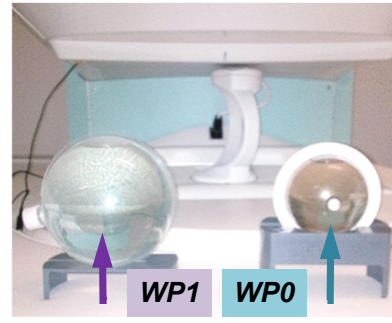

a)

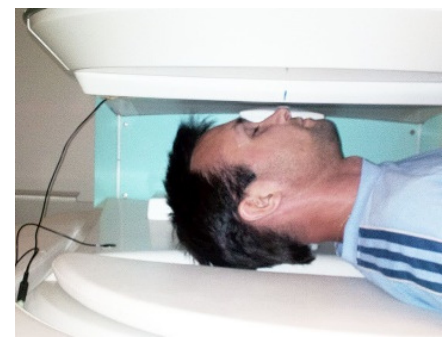

b)

Fig.13. Arrangement of the vibration signal pick up in the MRI Opera: using two test water phantoms a) with a lying person b), the vibration sensors SB-2a,b mounted in the left corner.

\section{DISCUSSION AND CONCLUSIONS}

In the first step, the acoustic noise was measured in the vicinity of the open-air MRI equipment E-scan Esaote Opera. The maximum sound pressure level of about $72 \mathrm{~dB}(\mathrm{C})$ was achieved for the sound level meter located in the direction of 90 degrees, at the height of $95 \mathrm{~cm}$ when the SE scan sequence with sagittal orientation was executed and the tested person was lying in the scanning area. Under the same circumstances, the background noise $S P L_{0}$ originating from the temperature stabilizer reached approximately $55 \mathrm{~dB}(\mathrm{C})$ when measured in the time instant with no scan sequence running. As shown in bar-graph in Fig.8., SPL increases at both heights (lower and higher gradient coils) when the examined person is present in between. 

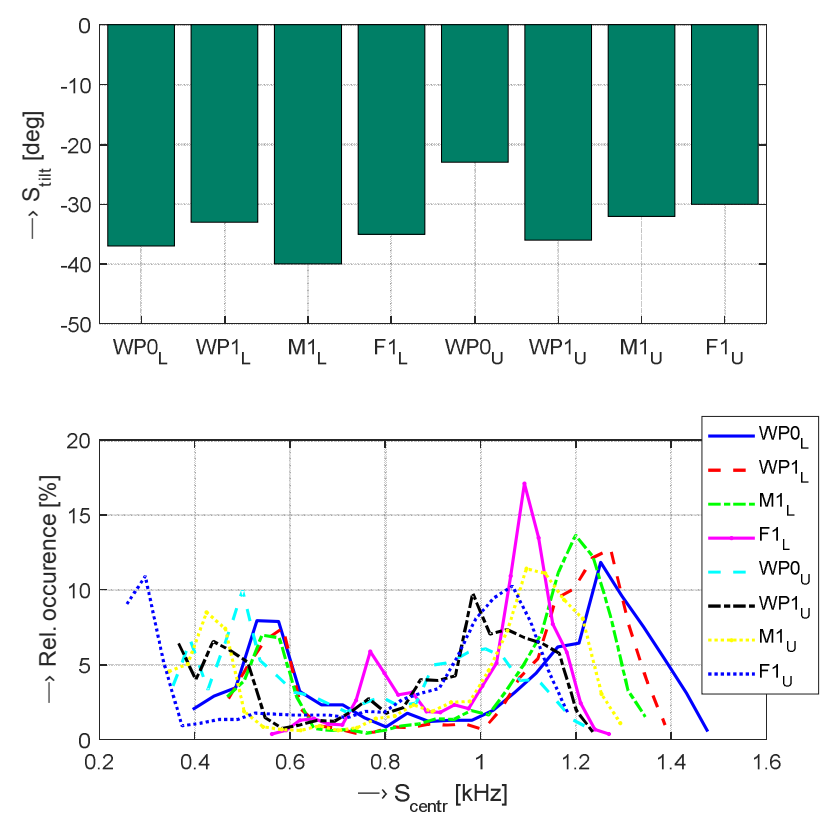
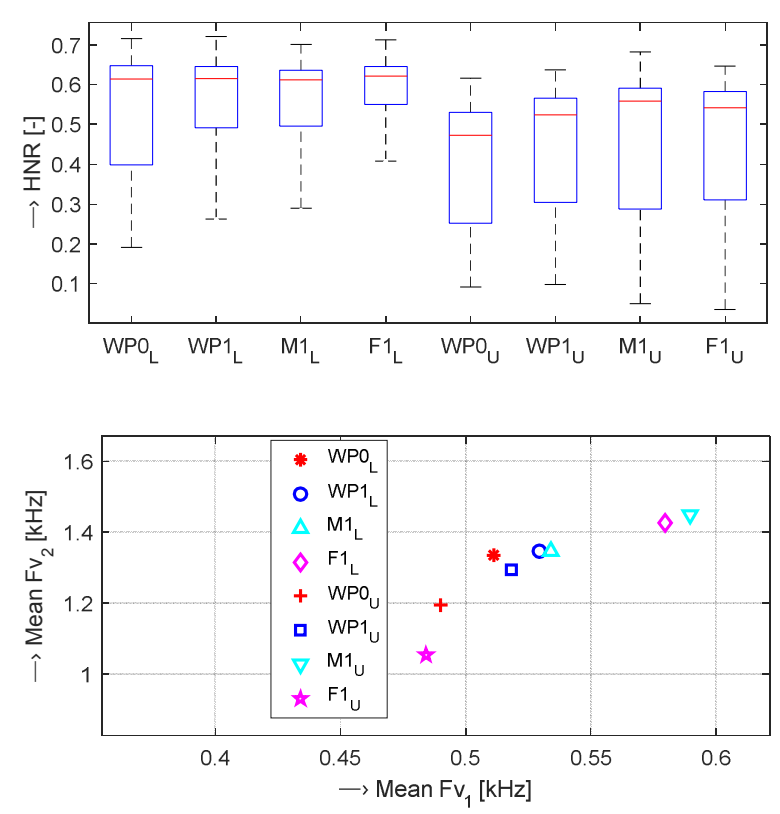

Fig.14. Comparison of spectral properties of the vibration signals for different subjects inserted in the scanning area of the MRI device: $\left\{\right.$ Spectral tilt, Spectral entropy, Spectral centroid, $F_{\mathrm{v} 1}$ to $F_{\mathrm{v} 2}$ mutual positions\}; SE scan sequence, sagittal orientation.

For the loaded lower cover of the gradient coils, SPL becomes higher at the upper gradient coils, e.g. for the water phantom it is about $8 \mathrm{~dB}$ higher than at the lower gradient coils. It may be caused by a greater effect of mechanical damping of the object lying on the lower cover than the effect of mass and mechanical compliance that would give rise to a new mechanical resonance.

On the other hand, the influence of the measuring direction is low - the standard deviation of SPL for directions of 30 , 90 , and 150 degrees is practically minimal. In general, the obtained noise SPL values were roughly inversely proportional to the effective weights of the lying male or female person.

The second part of our experiment was directed at processing and analysis of the vibration signals picked up simultaneously from the plastic cover of the lower and the upper gradient coils (see the arrangement photo in Fig.5.). The analysis of spectral differences between two mostly used MR scan sequences ( $\mathrm{SE} / \mathrm{GE}$ ) has shown that GE produces more structured vibration and SE generates more compact vibration as documented by comparison of HNR, spectral centroid, and $F_{\mathrm{V} 1}$ to $F_{\mathrm{V} 2}$ mutual positions in Fig.10.

Further investigation was aimed at the influence of the choice of slice orientation on the energy of the produced vibration signals. This effect is large - the maximum can be found in the sagittal plane and the minimum in the transversal plane - see the set of graphs in Fig.11. Therefore, the remaining experiments used only the sagittal orientation. While TR determines mainly the fundamental frequency $F_{\mathrm{V} 0}$, $\mathrm{TE}$ affects higher frequencies as documented by the graphs of spectral properties in Fig. 12.

The last part of the analysis of the recorded vibration signals confirms our basic assumption about the influence of the volume size placed in the scanning area of the MRI device on the vibration energy relations during the gradient field changes when the scanning MR sequence is executed. The obtained values of the signal RMS stored in Table 1. show twofold increase in the vibrational energy for the testing person (male/female) inside the MRI device when compared with the water phantom (WP0/WP1). On the other hand, this effect is manifested only in the vibration signal taken from the upper part of the gradient system. The opposite tendency is observed at the lower gradient coils - the signal RMS is higher for the testing phantom. This effect can be explained by higher mechanical damping of the system composed of the lower cover and the lying person than that of the lower cover with the sphere phantom. Both mentioned phenomena are evident in changes of the spectral properties in Fig.14. The values of HNR are higher for the lower part of the gradient system which is consistent with more resonances of the lower part comprising also the mass and the mechanical compliance of the object. The spectral centroid as well as the $F_{\mathrm{V} 1,2}$ frequencies are increased and this effect is more pronounced in the case of the vibration signals taken from the upper gradient coils.

The results of the performed measurements are useful for precise description of the process of mechanical vibration excitation and the acoustic noise radiation in the scanning area and the vicinity of the MRI device. Generalization can be made for the cases when it is difficult or impossible to get undistorted measurement results. The measurement results taken from a similar low field MRI tomograph can be used when the acoustic noise suppression in the recorded speech is necessary [17].

The realized calibration of the vibration sensor SB2a,b yielded some non-linearity in its frequency response for lower frequencies in the range of about $40-100 \mathrm{~Hz}$ as documented by the graph in Fig3.b). Therefore, frequency 
response linearization must be done during the signal preprocessing phase. A frequency equalizer as a combination of a low-pass shelve filter with a peak filter [19] can be used for this purpose. This linearization will have effect on correctness of all the spectral properties determined from the vibration signals.

\section{ACKNOWLEDGMENT}

This work was supported by the Slovak Scientific Grant Agency project VEGA 2/0001/17, the Ministry of Education, Science, Research, and Sports of the Slovak Republic VEGA $1 / 0905 / 17$, and within the project of the Slovak Research and Development Agency Nr. APVV-15-0029.

\section{REFERENCES}

[1] Esaote S.p.A. (2008). E-scan Opera. Image Quality and Sequences Manual. 830023522 Rev. A.

[2] Wang, F., Mechefske, C.K. (2008). Vibration analysis and testing of a thin-walled gradient coil model. Journal of Sound and Vibration, 311, 554-566.

[3] Winkler, S.A., Alejski, A., Wade, T., McKenzie, C.A., Rutt, B.K. (2017). On the accurate analysis of vibroacoustics in head insert gradient coils. Magnetic Resonance in Medicine, 78, 1635-1645.

[4] Latta, P., Gruwel, M.L.H., Edie, E., Šrámek, M., Tomanek, B. (2004). Single point imaging with suppressed sound pressure levels through gradientshape adjustment. Journal of Magnetic Resonance, 170, 177-183.

[5] Glowacz, A. (2015). Recognition of acoustic signals of synchronous motors with the use of MoFS and selected classifiers. Measurement Science Review, 15 (4), 167175.

[6] Winkler, S.A., Schmitt, F., Landes, H., de Bever, J., Wade, T., Alejski, A., Rutt, B.K. (2018). Gradient and shim technologies for ultra high field MRI. NeuroImage, 168, 59-70.

[7] Wang, Y., Liu, F., Li, Y., Tang, F., Crozier, S. (2016). Asymmetric gradient coil design for use in a short, open bore magnetic resonance imaging scanner. Journal of Magnetic Resonance, 269, 203-212.
[8] Weiger, M., Overweg, J., Rösler, M.B. et al. (2018). A high-performance gradient insert for rapid and short-T2 imaging at full duty cycle. Magnetic Resonance in Medicine, 79, 3256-3266.

[9] Přibil, J., Přibilová, A., Frollo, I. (2014). Mapping and spectral analysis of acoustic vibration in the scanning area of the weak field magnetic resonance imager. Journal of Vibration and Acoustics - Transactions of the ASME, 136 (5), 051005.

[10] Přibil, J., Přibilová, A., Frollo, I. (2016). Comparison of mechanical vibration and acoustic noise in the open-air MRI. Applied Acoustics, 105, 13-23.

[11] Luukinen, J.M., Aalto, D., Malinen, J. et al. (2018). A novel marker based method to teeth alignment in MRI. Measurement Science Review, 18 (2), 79-85.

[12] Diedrichsen, J., Balsters, J.H., Flavell, J., Cussans, E., Ramnani, R. (2009). A probabilistic MR atlas of the human cerebellum. NeuroImage, 46 (1), 39-46.

[13] Hamaguchi, T., Miyati, T., Matsushita, T., Ohno, N. (2014). Analysis of spatial dependence of acoustic noise transfer function in magnetic resonance imaging. In European Congress of Radiology (ECR 2014), March 6-10, 2014, Vienna, Austria.

[14] Liang, Z.P., Lauterbur, P.C. (1999). Principles of Magnetic Resonance Imaging: A Signal Processing Perspective. Wiley-IEEE Press.

[15] Fraden, J. (2010). Handbook of Modern Sensors: Physics, Designs, and Applications. Springer.

[16] Mechefske, C.K. (2008). Vibration in MRI scanners. In Biomedical Applications of Vibration and Acoustics in Therapy, Bioeffect And Modeling. ASME, 329-349.

[17] Přibil, J., Horáček, J., Horák, P. (2011). Two methods of mechanical noise reduction of recorded speech during phonation in an MRI device. Measurement Science Review, 11 (3), 92-98.

[18] Wei, J., Liu, J., Fang, Q., Lu, W., Dang, J., Honda, K. (2016). A novel method for constructing 3D geometric articulatory models. Journal of Signal Processing Systems, 82, 295-302.

[19] Dutilleux P., Zölzer, U. (2002). Filters. In DAFX Digital Audio Effects. John Wiley \& Sons, 31-62.

Received April 17, 2018 Accepted September 18, 2018 